\title{
Inhibitory Effect of Curcumin on Invasion of Skin Squamous Cell Carcinoma A431 Cells
}

\author{
Jian Wu${ }^{1}$, Wen-Ying $\mathrm{Lu}^{2 *}$, Lei-Lei Cui ${ }^{1 *}$
}

\begin{abstract}
Objective: To investigate the regulatory effect of curcumin on expression of signal transducer and activator of transcription 3 (STAT3) in skin squamous cell carcinoma tissues as well as possible mechanisms of curcumin in prevention and treatment of skin squamous cell carcinoma. Materials and Methods: Highly invasive A431 cells were treated with curcumin at various doses.The cytotoxic effects of treatment with $5,10,15,20,25,30,35$, 40 and $50 \mathrm{umol} / \mathrm{L}$ curcumin for 24,48 and 72 hours on A431 cells were measured by MTT assay. The invasion capacity of cells treated with 5,10 and $15 \mathrm{umol} / \mathrm{L}$ curcumin was measured by Transwell test, while adhesive ability was assessed by cell adhesion assay. The effects of 5,10 and $15 \mathrm{umol} / \mathrm{L}$ curcumin on expression levels of STAT3 were determined by Western blotting and on transcription levels of STAT3 mRNA by RT-PCR. Results: Treatment with curcumin at a doses of more than $15 \mathrm{umol} / \mathrm{L}$ for more than 24 hour inhibited the growth of A431 cells in a time-and dose-dependent fashion $(p<0.001)$. The doses of $15 \mathrm{umol} / \mathrm{L}$ and less for 24 hours showed no significant cytotoxic effects on the cells, survival rates being more than $85 \%$.The invasion and adhesive abilities decreased gradually with the increasing curcumin concentration, $15 \mathrm{umol} / \mathrm{L}$ exerting the strongest inhibitory effects $(p<0.05)$. Curcumin showed significant dose-dependent inhibitory effects on the transcription level of STAT3 mRNA $(p<0.05)$. Conclusions: Curcumin may reduce the invasive ability of A431 cells by inhibiting the activation of STAT3 signal pathway and expression of STAT3 as a target gene in the pathway.
\end{abstract}

Keywords: Curcumin - skin squamous cell carcinoma - signal transducer - activator of transcription 3(STAT3)

Asian Pac J Cancer Prev, 16 (7), 2813-2818

\section{Introduction}

In the past 30 years, the morbidity and mortality of cancer has been demonstrating a growing trend (Kraljik et al., 2014; Qian et al., 2014). Skin squamous cell carcinoma (SCC) is a higher incidence of skin malignant tumors, it has a large proportion in the skin malignant tumors, is commonly 80\% 90\% (Jian et al., 2014). incidence has increased year by year, especially in the elderly. Compared with the addition of a common skin basal cell carcinoma, squamous cell carcinoma of the skin of the illness development is rapid, destructive, but lymph node metastasis, visceral metastasis can be advanced, so people pay more and more attention to the prevention and control.

The treatment of Western medicine at present no effective and economic means, traditional Chinese medicine because of its extensive pharmacological effects and advantages of the side effects of smaller, in-depth study of traditional Chinese medicine and composition in cutaneous squamous cell carcinoma pathogenesis has important significance in genetic toxicity.

Signal transducer and activator of transcription factor 3 (STAT3) is a key molecule in various oncogenic signaling pathway, the downstream regulation of many growth, and tumor cell apoptosis, angiogenesis and metastasis related key points, such as CyclinD1, Survivin, Bcl-2, MMP2, STAT3 involved in cell growth (Chen et al., 2013), differentiation, division and development of many physiological process, and plays an important role in the malignant transformation of cells( Qu et al., 2013). STAT3 is the signal transducers and activators of transcription (STAT) is an important member of the family. STAT3, as a transcription factor in the absence of specific stimuli located in the cytoplasm, when cells are stimulated, domain and structure of STAT3 receptor $\mathrm{SH} 2$ on residues is tyrosine phosphorylated in combination, at the same time its phosphorylation by JAK (Talbot et al., 2014). STAT3 is EGFR (Aparicio-Siegmund et al., 2014), IL-6/JAK, Src etc (Liu et al., 2014). Focusing multiple oncogenic tyrosine kinase signal channel, in a variety of tumor cells and tissues have had multiple activation, abnormal expression of key genes in STAT3 after the induced activation of the cell proliferation and differentiation, survival, apoptosis, closely related, through various channels to promote cell proliferation, malignant transformation, impede apoptosis expression of carcinogenesis, so at present is defined as a cancer gene 
(Wang et al., 2014).

STAT3 not only highly expressed in many tumor cells, but also the continuous high expression in tumor microenvironment in a variety of cells, therefore, inhibit tumor cells and their micro-environment of various stromal cell STAT3 is often considered a target for tumor therapy.

Curcumin is a kind of natural plant polyphenol pigments, extracted from the Zingiberaceae turmeric, there are other Zingiberaceae plants. Widely exists in turmeric rhizome in traditional Chinese medicine. At present, curcumin has been proven to be a safe and effective inhibitors of STAT3, as in T cell leukemia, (Chung et al., 2015). curcumin can inhibit the STAT3 upstream of JAK kinase activity (Uehara et al., 2014), constitutively phosphorylated (Hu et al., 2014). To prevent STAT3, in skin squamous cell carcinoma, whether curcumin can inhibit STAT3 signaling pathway to prevent tumor invasion and metastasis, so far there is no clear reports. The experiments on the effects of different concentrations of curcumin on expression of STAT3 in skin squamous cell carcinoma A431 cells, further explore the mechanism of effect of skin squamous cell carcinoma invasion and metastasis.

\section{Materials and Methods}

\section{Cell culture}

The human skin squamous cell line A431 cells (Shanghai cell biology Institute, China.) were cultured in RPMI1640(GIBCO company, USA) containing 10\%FBS, and incubated under the condition of $37^{\circ} \mathrm{C}$ and $5 \% \mathrm{CO}_{2}$ in the cell culture box, when the cell growth fusioned to $80 \%$, they were digested with pancreatic digestive enzyme of $0.25 \%$.

\section{Detection of cell killing treated with curcumin}

Cells in the logarithmic phase were inoculated in 96 well plates by $4 \times 10^{3} /$ hole, the edge hole was added sterile PBS as control. Curcumin (Pharmaceutical and biological company,China) were added at the concentrations of 5, $10,15,20,25,30,35,40$ and $50 \mu \mathrm{mol} / \mathrm{L}$ after 24 hour. The experimental control group (0.1\%DMSO) and zero group were seted up, each group had 3 holes. Cultured for $24,48,72$ hour, they were centrifuged at $1000 \mathrm{r} / \mathrm{min}$, discarded the culture medium. Serum free RPMI1640 medium and 20ul MTT (Sigma, American) solution (5mg/ ml) were added in per hole with $180 \mu \mathrm{L}$. Cultured for 4 hour, they were centrifuged at $1000 \mathrm{r} / \mathrm{min}$, discarded the supernatant, $100 \mu \mathrm{L}$ DMSO were added in each hole , shaked for $10 \mathrm{~min}$. Optical density at A value was detected by wavelength standard instrument at the wavelength of $490 \mathrm{~nm}$, the cell survival rate (\%) was calculated according to the following formula.

Cell survival rate $(\%)=(\mathrm{A} 490($ experimental group $)-$ A490(control group)/ A490 (experimental group) $\times 100 \%$. The experiment was repeated 3 times.

Detection of invasion effect with curcumin by Transwell chamber invasion assay

Cells in the logarithmic growth phase were divided into 4 groups: negative control group (0.1\%DMSO) and curcumin $(5,10,15 \mathrm{umol} / \mathrm{L})$ treatment group. The cells were pretreated for 24 hour. The matrigel was melted overnight, then it was diluted with RPMI1640 culture medium without serum as 1:5, and was joined on the top of the Transwell chamber (Milliproe company) by 50ul/ hole (69.3ugMatrigel/hole), incubated in the incubator for 4 hour. The cells were digested with pancreatic enzyme of $0.25 \%$, were suspended with serum-free RPMI 1640 culture medium containing $0.2 \% \mathrm{BSA}$. The cells were adjusted to $5 \times 10^{5} / \mathrm{ml}$. The Transwell Chambers were added into the plate with 24 holes, cell suspension with 200ul were added into the top room, RPMI1640 culture medium containing $10 \%$ fetal bovine serum with $500 \mathrm{ul}$ were added into the lower room. They were incubated under the condition of $37^{\circ} \mathrm{C}$ and $5 \% \mathrm{CO}_{2}$ in the cell culture box for 24 hour. After being dried, they were stained with $0.1 \%$ crystal violet for $10 \mathrm{~min}$, washed 3 times with PBS, fixed with $95 \%$ ethanol. Five different field of vision were selected randomly of each chamber, cells were counted under light microscope with 100 times. The mean and variance were calculated.

\section{Detection of adhesion ability by adhesion test}

Cells in the logarithmic growth phase were divided into 4 groups: negative control group (0.1\%DMSO) and curcumin $(5,10,15 \mathrm{umol} / \mathrm{L})$ treatment group. The cells were pretreated for 24 hour. The 96-well plate was packaged with fibronectin solution, which were prepared to a final concentration of $10 \mathrm{ug} / \mathrm{ml}$ with filter double distilled water. It was saved at $4^{\circ} \mathrm{C}$ overnight after being sealed. It was washed 3 times by PBS. Serum-free RPMI1640 culture medium containing $0.2 \%$ BSA were added into each hole of $50 \mathrm{ul} / \mathrm{hole}$. It was closed for 2 hour at $37^{\circ} \mathrm{C}$. The cells were digested with pancreatic enzyme of $0.25 \%$, were suspended with serum-free RPMI 1640 culture medium containing $0.2 \%$ BSA. The cells were adjusted to $2 \times 10^{4}$ /hole. The cells were vaccinated in 96well plates, each group was settled up for 5 holes. The 96-well plates were incubated in cell cultured box for 1 hour at $37^{\circ} \mathrm{C}$. After being incubated, they were washed for 3 times by PBS, which was warmed in advance. MTT $(2 \mathrm{mg} / \mathrm{ml})$ was added into each hole by $50 \mathrm{ul} / \mathrm{hole}$. Then it was incubated in cell cultured box for 3 hour at $37^{\circ} \mathrm{C}$. After being incubated, MTT was discarded, DMSO was added into each hole with $150 \mathrm{ul} /$ hole. Being shaked for 10min, the Optical density at A value was detected by wavelength standard instrument at the wavelength of 490nm, the cell adhesion amount was calculated according to the following formula.

Cell adhesion amount $=(\mathrm{A} 490($ experimental group $)-$ A490(control group).

\section{Detection of the expression of STAT3, p-STAT3 by using Western blot}

A431 cells were inoculated into the culture bottle by $1 \times 10^{4} / \mathrm{cm}^{2}$. After being adhereded, the medicines were added into the bottle respectively, according to the following group: the negative control group (0.1\% DMSO) and curcumin $(5,10,15 \mathrm{umol} / \mathrm{L})$ treatment group. After being incubated for $24 \mathrm{~h}$, the total protein of the cells were extracted. It was separated by $10 \%$ SDS-PAGE, then it 
Table 1. Primer Sequences for RT-PCR Analysis

\begin{tabular}{lllc}
\hline Primers & Primer sense & Primer sequences 5' ${ }^{\prime}{ }^{\prime}$ & Product size \\
\hline STAT3 & Forward & CCTTCCTGCGGTTCAGT & $344 \mathrm{bp}$ \\
& Reverse & GCTGCAGGTCGTTGGTGCTAC & $344 \mathrm{bp}$ \\
$\beta$-action & Forward & ATCGTGCGTGACATTAAGGAGAAG & $231 \mathrm{bp}$ \\
& Reverse & AGGAAGGAAGGCTGGAAGTC & $231 \mathrm{bp}$ \\
\hline
\end{tabular}

was transferred onto PVDF membrane. The membrane was closed by $5 \%$ skim milk at room temperature for $1 \mathrm{~h}$. Then it was washed by TBST for 3 times $(5 \mathrm{~min} /$ time). The first antibody of STAT3 mouse monoclonal antibody and P-STAT3 mouse monoclonal antibody (Cell Signaling Technologies company,USA)was added on the membrane, it was incubated at $4{ }^{\circ} \mathrm{C}$ overnight. It was washed by TBST for 3 times $(5 \mathrm{~min} /$ time $)$ again. The secend antibody was added on the membrane, shaked for $1 \mathrm{~h}$ at room temperature. Then it was washed by TBST for 3 times $(5 \mathrm{~min} /$ time $)$. It was imaged by ECL imaging system.

Detection of transcription level of STAT3 gene by RT-PCR

Cells in the logarithmic phase were inoculated in 6-well plates by $2 \times 10^{5} / \mathrm{cm}^{2}$. After being adhered, the medicines were added into the bottle respectively, according to the following group: the negative control group (0.1\%DMSO) and curcumin $(5,10,15 \mathrm{umol} / \mathrm{L})$ treatment group. After being incubated for $24 \mathrm{~h}$, the total RNA of the cells were extracted. The concentration of total RNA was detected, 10ug of total RNA was reverse transcripted into cDNA by ReverTraAce- alpha -RT-PCR kit (TOYOBO company, China).The volume of total system was $20 \mathrm{ul}$, with the condition of $99^{\circ} \mathrm{C}$ for $5 \mathrm{~min}, 4^{\circ} \mathrm{C}$ for $5 \mathrm{~min}$. The cDNA was stored at $-20^{\circ} \mathrm{C}$. The sequences of primers(SANGON, China) were showed in Table 1. Beta action gene was used as an internal matter.

Thermal cycling conditions were as follows: predenaturation at $94^{\circ} \mathrm{C}$ for $3 \mathrm{~min} ; 26$ cycles of $94^{\circ} \mathrm{C}$ for 30 $\mathrm{s}, 55^{\circ} \mathrm{C}$ for $30 \mathrm{~s}$, and $72^{\circ} \mathrm{C}$ for $2 \mathrm{~min}$, and re-extension at $72^{\circ} \mathrm{C}$ for $10 \mathrm{~min}$. PCR amplification products were separated on $2 \%$ agarose gel. The imaging acquisition was collected by BIO-RAD system, and analyzed by Quantity One software.

\section{Statistical methods}

SPSS13.0 statistical software was used for statistical analysis. Data are given as mean and standard deviation (Mean \pm SD). Between the two groups were compared using independent samples T-test, single factor analysis of variance for comparison of multiple groups of data, with $p<0.05$ for the differences have statistical significance.

\section{Results}

\section{The cell viability of MTT detection}

When the concentration of curcumin is more than 15 umol/L, the action time was more than $24 \mathrm{~h}$, the growth inhibition of cells in a time and dose dependence, and the difference was statistically significant $(p<0.001)$; when the concentration of curcumin is less than or equal to $15 \mathrm{umol} / \mathrm{L}$, the reaction time was $24 \mathrm{~h}$, there is no obvious toxic effect on the cells, the cell survival rate $>85 \%$ ( Table
Table 2. The Concentration Gradient of Curcumin Inhibition of A431 Cells after rate $(x \pm s, n=3)$

\begin{tabular}{|c|c|c|c|}
\hline \multicolumn{2}{|c|}{$\begin{array}{l}\text { Group } \\
\text { (umol/L) }\end{array}$} & \multicolumn{2}{|l|}{ Inhibition(\%) } \\
\hline & $24 \mathrm{~h}$ & $48 \mathrm{~h}$ & $72 \mathrm{~h}$ \\
\hline 5 & $4.30 \pm 3.30$ & $16.66 \pm 4.49$ & $27.36 \pm 3.18$ \\
\hline 10 & $12.30 \pm 0.53 \#$ & $32.11 \pm 2.51 \#$ & $56.73 \pm 0.31^{\# \bullet \bullet \bullet}$ \\
\hline 15 & $20.62 \pm 0.62^{\nabla}$ & $54.12 \pm 2.12^{\nabla}$ & $61.23 \pm 2.48^{\triangle} \bullet$ \\
\hline 20 & $63.33 \pm 0.84 *$ & $71.79 \pm 0.64 * \square$ & $75.00 \pm 1.09^{\triangle} \bullet$ \\
\hline 25 & $61.11 \pm 1.27 *$ & $69.65 \pm 0.98 *$ & $67.57 \pm 10.98^{\triangle}$ \\
\hline
\end{tabular}

*vs 5,10,15umol/L, $p<0.05 ;$ vs $5,10 \mathrm{umol} / \mathrm{L}, p<0.05 ; v s 5,10,20,25 \mathrm{umol} / \mathrm{L}$, $p<0.05$; \#vs 5,15,20,25umol/L, $p<0.05$; vs $24 \mathrm{~h}, p<0.05 ;$; $s$ 48h, $p<0.05$
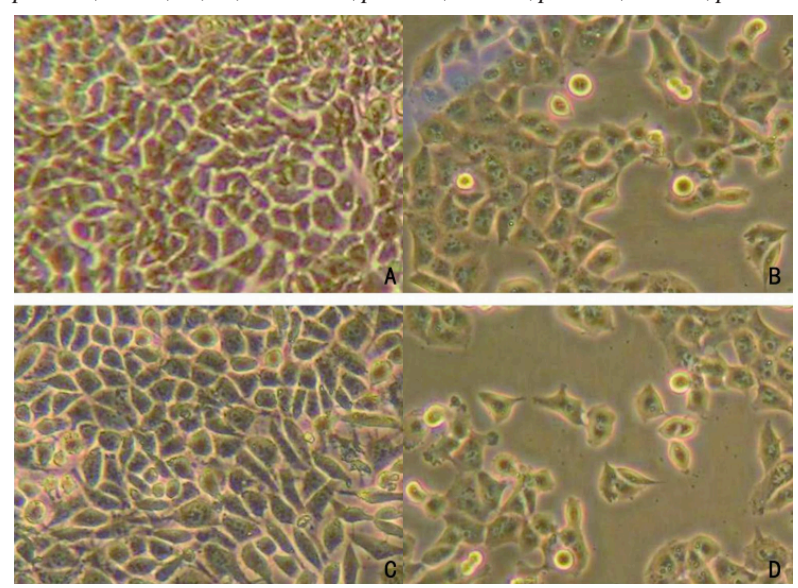

Figure 1. Effect of Curcumin on the Invasion Ability of A431 Cells in vitro ( $\times 100)$. A) negative control group $(0.1 \% \mathrm{DMSO})$; B) curcumin $(5, \mathrm{umol} / \mathrm{L})$ treatment group; $\mathbf{C})$ curcumin $(10 \mathrm{umol} / \mathrm{L})$ treatment group; D) curcumin $(15 \mathrm{umol} / \mathrm{L})$ treatment group

2). the choice the concentration of curcumin is less than 15 umol/L and the action time is less than or equal to $24 \mathrm{~h}$ are selected for the test conditions of invasion and adhesion.

\section{The invasive ability of cells}

The numbers of transmembrane cell are $(472.33+37.17)$, $(309.33+38.99),(187+17.06)$ and $(126.33+8.62) /$ vision of the negative control group and curcumin $(5,10$, $15 \mathrm{umol} / \mathrm{L}$ ) treatment group (Figure 1). Compared with the negative control group, the number of transmembrane cell gradually decreased with the drug concentration of curcumin gradually increased, the treatment by $15 \mathrm{umol} / \mathrm{L}$ has the most obvious effect. The differences between each group was statistically significant $(\mathrm{F}=85.66, p<0.05)$.

\section{Cell adhesion ability}

The amount of cell adhesion are $(1.357+0.094)$, $(0.878+0.026),(0.482+0.032)$ and $(0.3036+0.030)$ of the negative control group and curcumin $(5,10,15 \mathrm{umol} / \mathrm{L})$ treatment group. Compared with the negative control group, the ability of cell adhesion gradually decreased with 


\section{Jian Wu et al}

the drug concentration of curcumin gradually increased, the treatment by $15 \mathrm{umol} / \mathrm{L}$ has the most obvious effect. The differences between each group was statistically significant $(\mathrm{F}=381.74, p<0.01)$.

\section{Expression of STAT3, P-STAT3 protein}

The result of Western blot shows that the inhibition of the expression with P-STAT3 gradually enhanced, while the the concentration of curcumin gradually increased. It is in a dose dependent. The treatment by $15 \mathrm{umol} / \mathrm{L}$ has the most obvious effect. The differences between each group was statistically significant $(\mathrm{F}=116.07, p<0.05)$, while there is no significant difference in the level of STAT3 protein (Figure 2 and Figure 3).

\section{STAT3 gene transcription level of $m R N A$}

The results of RT-PCR show that the inhibition of the transcription level of STAT3 gradually enhanced, while the the concentration of curcumin gradually increased. It is in a dose dependent. The transcription level of STAT3 are $(0.781+0.135),(0.515+0.174),(0.268+0.062),(0.045+$ $0.003)$ of the negative control group and curcumin (5, $10,15 \mathrm{umol} / \mathrm{L})$ treatment group(Figure 4). Compared with the negative control group, the transcription level gradually decreased with the drug concentration of curcumin gradually increased, the treatment by 15 umol/L has the most obvious effect $(\mathrm{t}=9.445, p<0.01)$. The differences between each group was statistically significant $(\mathrm{F}=23.022, p<0.05)$.

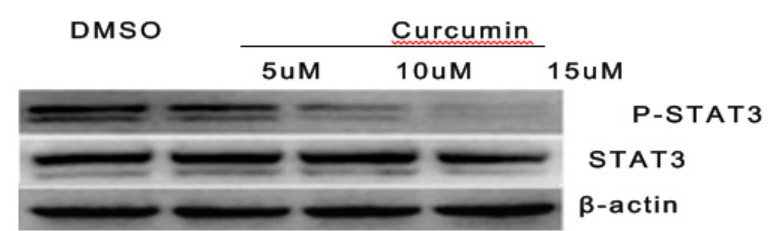

Figure 2. Effect of Curcumin on Expression of STAT3 and P-STAT3 Protein in A431 Cells

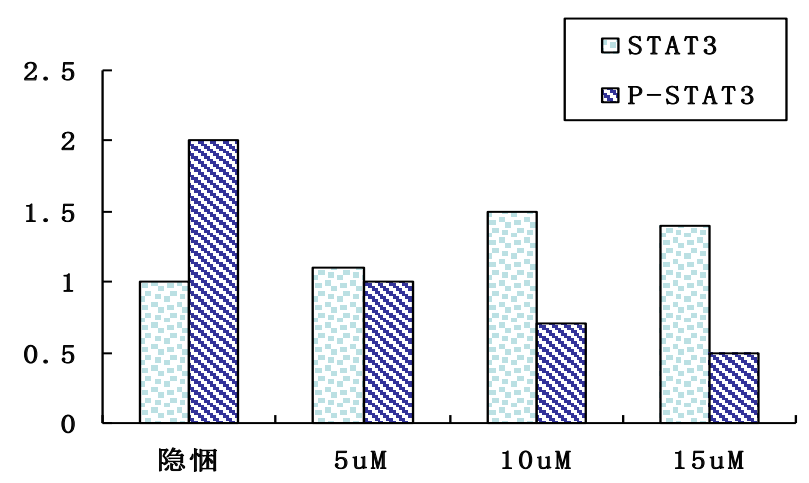

Figure 3. The Relative Expression of STAT3 and P-STAT3 Protein in A431 Cells Western Blot detection after Curcumin Treatment

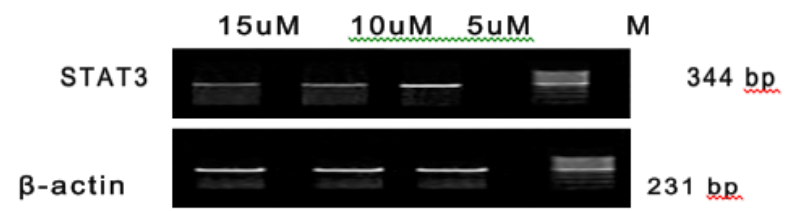

Figure 4. Effect of Curcumin on STAT3 Cells A431 Gene Transcription Level of mRNA

\section{Discussion}

In the past 30 years, China's cancer disease incidence rate and the mortality rate has been a growing trend. Cutaneous squamous cell carcinoma is higher incidence rate of malignant skin tumors, which occupies a great proportion in the skin malignant tumor occurrence and ultraviolet radiation, the skin squamous cell carcinoma is closely related with the environment, and the destruction of the ozone layer increased, its incidence has been rising trend, generally $80 \%$ to $90 \%$, especially in the elderly. With the addition of a common skin basal cell carcinoma (basal, cell carcinoma, BCC) compared with squamous cell carcinoma of the skin, illness development is rapid, destructive, but lymph node metastasis, visceral metastasis can be advanced, so people pay more and more attention to the prevention and control. The treatment of Western medicine at present no effective and economic means, traditional Chinese medicine because of its extensive pharmacological effects and advantages of the side effects of smaller, in-depth study of traditional Chinese medicine and its components in skin squamous cell carcinoma pathogenesis has important significance in genetic toxicity. In recent years there has been a lot of research shows that many Chinese traditional medicine has strong antitumor effect, and in the induction of apoptosis, promoting differentiation and improve immune function has its unique advantage. Jiang Huang is commonly used in traditional Chinese medicine, and its main bioactive components for thecurcuminoids and essential oil. The former has the function of reducing blood lipid, antioxidant, anticoagulation, choleretic, anticancer and so on; the latter mainly anti-inflammatory, antibacterial and cough relieving effect. As a kind of anticancer drugs with good development prospect, has become a hot spot of research. Recent studies showed that curcumin, in animal models of skin cancer, liver cancer, colon cancer, lung cancer, ovarian cancer, breast cancer, gastric cancer and other tumor cells have obvious inhibition, and induce apoptosis of tumor cells. Current studies suggest that curcumin, by inducing malignant tumor cell differentiation (Kang et al., 2015), inducing apoptosis of tumor cells (Toden et al., 2015). and exerts its anticancer effect (Fiala et al., 2015). On tumor growth inhibitory effect of each period, a wide range of clinical applications (Zhou et al., 2015).

At present, curcumin has been proven to be a safe and effective inhibitors of STAT3, as in T cell leukemia, curcumin can inhibit the STAT3 upstream of JAK kinase activity, constitutively phosphorylated to prevent STAT3, in H-RS cells, curcumin can inhibit the phosphorylation of JAK2 through to block phosphorylation STAT3, STAT3 is the signal transducers and activators of transcription (STAT) is an important member of the family. STAT3. Focusing multiple oncogenic tyrosine kinase signal channel, in a variety of tumor cells and tissues have had multiple activation, abnormal expression of key genes in STAT3 after the induced activation of the cell proliferation and differentiation, survival, apoptosis, closely related, through various channels to promote cell proliferation, malignant transformation, impede apoptosis expression 
of carcinogenesis, so at present is defined as a kind of oncogene (Kim et al., 2013), STAT3 not onlyhighly expressed in many tumor cells (Xu et al., 2014), but also the continuous high expression in tumor micro-environment in a variety of cells, therefore, inhibit tumor cells and their micro-environment of various stromal cell STAT3 is often considered a target for tumor therapy. In order to clarify the curcumin through inhibition of STAT3 signaling pathway and inhibition of STAT3 gene expression on the invasion of skin squamous cell carcinoma A431 cells, Several signaling channel of carcinogenic tyrosine kinase converge into the point of STAT3, such as EGFR, IL-6/JAK, Src, and the over activation occurs in diverse kinds of cancer cells and tissues. After the activation, STAT3 induces this anomalous expression of pivotal gene related to cell activities, then speeds up cell proliferation and vicious transformation, inhibit anti-carcinogenesis function expressed by apoptosis by the various ways, therefore, it is descripted as a kind of oncogene (Snyder Met al., 2014). Our research shows that compared with normal skin tissues, positive rate of SCC on skin of phosphorylated STAT3 is raised obviously, and expression in SCC are all in nuclear type, which implies that the abnormal activation of STAT3 cascaded ingredient plays a significant role in the occurrence of skin SCC. The staining intensity of $p$-STAT3 in skin SCC of level II and III notably increases, which lead to the speculation that the over activation of STAT3 may be closely relevant to invasive growth of SCC.

In order to clarify the curcumin through inhibition of STAT3 signaling pathway and inhibition of STAT3 gene expression on the invasion of skin squamous cell carcinoma A431 cells, in this study, high invasive cutaneous squamous cell carcinoma cell line A431 as the research object, using different doses of curcumin treatment, we examined the influence of Jiang Huang element to the skin squamous cell carcinoma tumor cells invasion. The study of toxic effect on the cells by the method of MTT to detect different concentrations of curcumin in cells, affect the Transwell chamber invasion assay method for the detection of different concentrations of curcumin after cell invasion ability, ability to detect the change of cell adhesion adhesion experiments, results showed curcumin concentration, $15 \mathrm{umol} / 24 \mathrm{~h}$, the role of time, the growth inhibition of cells in a time and dose dependence, when curcumin concentration less than $15 \mathrm{umol} / \mathrm{L}$, the reaction time was 24 hour, no obvious toxic effect on the cells, the cell survival rate $>85 \%$; with the increase of the concentration of curcumin, cell invasion and adhesion ability decreased gradually with concentration of $15 \mathrm{umol} / \mathrm{L}$, the most obvious effect of curcumin effect.

This study using the Western blot method to detect the effect of different concentration of cells after STAT3 and P-STAT3 protein levels, but also by real-time fluorescent quantitative PCR detection effect of curcumin in different concentration plant cells after STAT3 gene transcription level of mRNA, indicated that curcumin could significantly inhibit the STAT3 gene transcription level of mRNA, the inhibition was dose dependence of it.

The study found that, the activation of STAT3 in skin squamous cell carcinoma (P-STAT3), promoting tumor cell invasion and metastasis, cyclin D1 STAT3 as a downstream gene expression, reported that cyclin D1 is to influence the process of invasion skin squamous carcinoma cells regulates the activation of STAT3. The research group through inhibition of P-STAT3 expression by curcumin, interference of $p$-STAT3 signal transduction pathway in cutaneous squamous cell carcinoma, attempts to prevents the $p$-STAT3 signal transduction pathway induced by over expression of cyclinD1 resulting in skin squamous cell carcinoma effect generated at the source, which will be anti tumor for clinical drug therapy and gene therapy offers a possible interference, open a new important way.

In summary, curcumin can significantly weaken the skin squamous cell carcinoma A431 cells by inhibiting expression of the activity of the STAT3 signaling pathway and the pathway target gene STAT3 on the invasion and metastasis ability.

\section{References}

Aparicio-Siegmund S, Sommer J, Monhasery N, et al (2014). Inhibition of protein kinase II (CK2) prevents induced signal transducer and activator of transcription (STAT) 1/3 and constitutive STAT3 activation. Oncotarget, 5, 2131-48.

Chen J, Chi M, Chen C, et al (2013). Obesity and melanoma: exploring molecular links. J Cell Biochem, 114, 1955-61.

Chung SS, Vadgama JV, et al (2015). Curcumin and epigallocatechin gallate inhibit the cancer stem cell phenotype via down-regulation of STAT3-NFxB signaling. Anticancer Res, 35, 39-46.

Dai XZ, Yin HT, Sun LF, et al (2013). Potential therapeutic efficacy of curcumin in liver cancer. Asian Pac J Cancer Prev, 14, 3855-9.

Fiala M(2015). Curcumin and omega-3 fatty acids enhance NK cell-induced apoptosis of pancreatic cancer cells but curcumin inhibits interferon- $\gamma$ production: benefits of omega-3 with curcumin against cancer. Molecules, 20, 3020-6.

$\mathrm{Hu}$ A, Huang JJ, Jin XJ, et al (2014). Curcumin suppresses invasiveness and vasculogenic mimicry of squamous cell carcinoma of the larynx through the inhibition of JAK-2/ STAT-3 signaling pathway. Am J Cancer Res, 5, 278-88.

Jian Wu, Jin-Rong Zhang, Jie Qin, et al (2014). Methylation of E-cadherin and p14ARF gene promoters. Int J Clin Exp Med, 7, 1808-12.

Kang JH, Kang HS, Kim IK, et al (2015). Curcumin sensitizes human lung cancer cells to apoptosis and metastasis synergistically combined with carboplatin. Exp Biol Med (Maywood).

Kim GT, Lee SH, Kim YM, et al (2013). Quercetin regulates sestrin 2-AMPK-mTOR Signaling pathway and induces apoptosis via increased intracellular ROS in HCT116 colon cancer cells. J Cancer Prev, 18, 264-70.

Kraljik N, Rosso M, Ageel A, et al (2014). The incidence of skin squamous cell carcinoma in Osijek-Baranja County--an epidemiological study. Coll Antropol, 35, 77-80.

Liu L, Gaboriaud N, Vougogianopoulou K, et al (2014). MLS2384, a new 6-bromoindirubin derivative with dual JAK/ Src kinase inhibitory activity, suppresses growth of diverse cancer cells. Cancer Biol Ther, 15, 178-84

Talbot JJ, Song X, Wang X, et al (2014). The cleaved cytoplasmic tail of polycystin-1 regulates Src-dependent STAT3 activation. J Am Soc Nephrol, 25, 1737-48. 


\section{Jian Wu et al}

Qian YD, Xu X, Wang L, et al (2014).Clinical safety of chemotherapy for elderly cancer patients complicated with hypertension. Asian Pac J Cancer Prev, 15, 9875-7.

Qu Y, Dang S, Hou P, et al (2013). Gene methylation in gastric cancer. Clin Chim Acta, 23, 53-65.

Snyder M, Huang J, Huang XY, et al (2014). A STAT3/NFxB complex is necessary for the expression of fascin in metastatic breast cancer cells in response to IL- 6 and TNF- $\alpha$. $J$ Biol Chem, 11, 30082-9.

Toden S, Okugawa Y, Buhrmann C, et al (2015). Novel evidence for curcumin and boswellic acid induced chemoprevention through regulation of miR-34a and miR-27a in colorectal cancer. Cancer Prev Res. [Epub ahead of print]

Uehara Y, Inoue M, Fukuda K, et al (2014). Inhibition of $\beta$-catenin and STAT3 with a curcumin analog suppresses gastric carcinogenesis in vivo. Gastric Cancer. [Epub ahead of print]

Wang J, Zhang L, Chen G, et al (2014). Small molecule 1 '-acetoxychavicol acetate suppresses breast tumor metastasis by regulating the SHP-1/STAT3/MMPs signaling pathway. Breast Cancer Res Treat. [Epub ahead of print]

Xu W, Yang Z, Zhou SF, et al (2014). Posttranslational regulation of phosphatase and tensin homolog (PTEN) and its functional impact on cancer behaviors, Drug Des Devel Ther, 8, 1745-51.

Zhou GZ, Sun GC, Zhang SN, et al (2015). The interplay between autophagy and apoptosis induced by one synthetic curcumin derivative hydrazinobenzoylcurcumin in A549 lung cancer cells. J Biochem Mol Toxicol. [Epub ahead of print] 